\title{
Fat Embolism Syndrome: Case Report
}

\author{
Sreenivasa Rao Sudulagunta ${ }^{\mathrm{a}, \mathrm{g}}$, Monica Kumbhat ${ }^{\mathrm{b}}$, Mahesh Babu Sodalagunta ${ }^{\mathrm{c}}$, \\ Aravinda Settikere Nataraju ${ }^{\mathrm{d}}$, Mona Sepehrare, \\ Shiva Kumar Bangalore Raja ${ }^{\mathrm{f}}$
}

\begin{abstract}
Fat embolism syndrome (FES) is a systemic inflammatory cascade affecting multiple organ systems occurring after trauma, orthopedic procedures and rarely in non-traumatic patients causing high morbidity and mortality. Fat emboli develop in many patients with bone fractures (incidence of this problem can be up to $90 \%$ in patients who have sustained major injuries), but are usually asymptomatic. A minority of patients develop signs and symptoms of organ system dysfunction due to mechanical obstruction of capillaries by fat emboli or due to fat hydrolyzing to fatty acids. A triad of lung, brain and skin involvement develops after 24 - 72 hours of asymptomatic period. This symptom complex is known as FES. Fat embolism is diagnosed clinically with non-specific and insensitive diagnostic tests. Treatment of FES is supportive and in most cases can be prevented by early fixation of large bone fractures. Here we report a case of traumatic fat embolism, treated successfully with supportive management. Diagnosis of FES needs high index of suspicion and use of clinical criteria along with imaging.
\end{abstract}

Keywords: Fat embolism syndrome; Magnetic resonance imaging; Trauma; Femur fracture; ARDS

\section{Introduction}

Ernst Von Bergmann, in 1873, was the first person that made a clinical diagnosis of fat embolism. He did this on the basis of 10 years knowledge gathered from experiments with cats, in which he injected intravenous oils. Von Bergmann also described a patient who had fallen off a roof and sustained a

Manuscript accepted for publication November 23, 2016

${ }^{a}$ Columbia Asia Hospital, Kirloskar Business Park, Hebbal, Bangalore, India bri Ramachandra Medical College, No. 1, Ramachandra Nagar, Porur, Chennai, Tamil Nadu 600116, India

${ }^{\mathrm{c}} \mathrm{K}$. S. Hegde Medical College, Mangalore, India

${ }^{\mathrm{d}}$ Columbia Asia Hospital, Hebbal, Bangalore, India

eBaptist Hospital, Bellary Road, Hebbal, Bangalore 560024, India

fDepartment of General Medicine, Dr. B. R. Ambedkar Medical College, Bangalore, India

gCorresponding Author: Sreenivasa Rao Sudulagunta, Columbia Asia Hospital, Kirloskar Business Park, Hebbal, Bangalore 560024, India.

Email: dr.sreenivas@live.in

doi: https://doi.org/10.14740/jnr405w comminuted fracture of the distal femur; $60 \mathrm{~h}$ after the injury, he developed dyspnea, cyanosis, and coma [1]. The common causes of fat embolism syndrome (FES) are represented in Table 1 . FES accounts for only $2-5 \%$ of patients who have longbone fractures [2]. In different studies, the FES incidence ranges from $<1 \%$ to $29 \%$, but the exact incidence is not clear $[3,4]$.

Symptoms of fat embolism occur 12 - $36 \mathrm{~h}$ after a traumatic injury usually [5]. Pulmonary dysfunction (dyspnea, tachypnea, and hypoxemia) is common, occurring in up to $75 \%$ of cases. About $10 \%$ of patients may develop respiratory failure and $5-8 \%$ may progress to acute respiratory distress syndrome (ARDS). Patients with bilateral fractures have higher incidence of ARDS compared to single fractures, reaching approximately $43 \%$ [6]. About $50 \%$ of FES patients require mechanical ventilation due to severe hypoxemia and respiratory insufficiency. Nevertheless, the role of fat emboli causing ARDS after injury has not yet been clearly defined.

The most common classification used for diagnosis of FES is by Gurd and Wilson; diagnosis of FES requires the presence of at least one major and four minor criteria [7] (Table 2). Schonfeld et al suggested a scoring system for FES diagnosis [8] (Table 3). Lindeque et al proposed that FES can be diagnosed based on respiratory changes [9] (Table 4). None of these criteria have been universally accepted.

\section{Case Report}

A 68-year-old female was brought to the emergency room after a fall while walking in the living room. The patient suffered

Table 1. Common Causes of Fat Embolism Syndrome

Blunt trauma (approximately $90 \%$ of all cases)

Acute pancreatitis

Diabetes mellitus

Burns

Joint reconstruction

Cardiopulmonary bypass

Liposuction

Decompression sickness

Sickle cell crisis

Parenteral lipid infusion

Pathologic fractures 
Table 2. Gurd and Wilson's Criteria

\begin{tabular}{ll}
\hline Major features & Minor features \\
\hline Axillary or subconjunctival petechiae & Tachycardia $>110 / \mathrm{min}$ \\
Hypoxemia $\mathrm{PaO}_{2}<60 \mathrm{~mm} \mathrm{Hg} ; \mathrm{FIO}_{2}=0.4$ & Pyrexia $>38.5$ \\
Pulmonary edema & Retinal fat or petechiae \\
Sudden drop in $\mathrm{Hb}$ level $>20 \%$ & Urinary fat globules or oligoanuria \\
$\begin{array}{l}\text { Central nervous system depression disproportionate } \\
\text { to hypoxemia }\end{array}$ & Sudden thrombocytopenia $>50 \%$ \\
\hline
\end{tabular}

ESR: erythrocyte sedimentation rate.

fracture of right inferior pubic ramus (Fig. 1) and was stable hemodynamically, without any alteration of sensorium. Chest radiography and arterial blood gas analysis were normal. $\mathrm{Pa}$ tient was opted for conservative management in view of comorbidities and option chosen by relatives. Twenty-four hours after admission, the patient developed decreased level of consciousness, hypotension and shortness of breath, manifested as tachycardia ( $>120 / \mathrm{min})$, dyspnea, and hypoxemia $\left(\mathrm{PO}_{2}: 70\right.$ $\mathrm{mm} \mathrm{Hg}, \mathrm{PaCO}_{2}: 39 \mathrm{~mm} \mathrm{Hg}, \mathrm{pH}$ 7.40).

Magnetic resonance imaging of brain with head and neck angiography showed multiple small focal acute infarcts in bilateral subcortical and paraventricular regions of frontoparietal lobes involving the corona radiata and centrum semiovale, suggestive of fat embolism (Figs. 2-4). A repeat chest radiograph showed bilateral perihilar fullness and features of ARDS (Fig. 5). Based on the patient's oxygen requirement and continued tachycardia and tachypnea, D-dimer assay was done and two values were found to be abnormal (1,600 and 1,750 $\mu \mathrm{mg} / \mathrm{L})$.

The patient was administered heparin and furosemide. Approximately $4 \mathrm{~h}$ later, the patient's oxygen saturations improved, from $79 \%$ to $91 \%$ on $5 \mathrm{~L}$ of oxygen. Her pulse returned to 104 beats per minute, and her systolic blood pressure stabilized in the range of 110- $125 \mathrm{~mm} \mathrm{Hg}$. Patient improved over a period of next $36 \mathrm{~h}$ with decreased need of oxygen. A review of her symptoms indicated the patient met five of Gurd and Wilson's criteria for FES, including petechiae, hypoxemia, pyrexia, tachycardia, and relative thrombocytopenia.

\section{Discussion}

The pathogenesis of FES is still controversial with several theories having been proposed [10]. Fat emboli pass through

Table 3. Schonfeld Criteria

\begin{tabular}{ll}
\hline Criteria & Score \\
\hline Petechiae & 5 \\
X-ray chest diffuse infiltrates & 4 \\
Hypoxemia & 3 \\
Fever & 1 \\
Tachycardia & 1 \\
Confusion & 1 \\
\hline
\end{tabular}

the pulmonary vasculature, resulting in systemic embolization, commonly affecting the brain and kidneys. As per the mechanical theory, FES occurs due to large fat globules entering the venous circulation resulting in the obstruction of the pulmonary vascular system. But, mechanical theory cannot clearly explain the delay in the development of symptoms. The biochemical theory states that hormonal changes after extensive trauma induce the hydrolysis of triglycerides and release of free fatty acids, causing toxic endothelium damage in pulmonary capillary beds, as well as ARDS in animal models. The time required to produce these toxic intermediaries can be explained to cause the delay in development of symptoms.

Cerebral manifestations of FES are variable and not specific: headache, irritability, lethargy, stupor, delirium, convulsions, or coma. Many cases remain undiagnosed as they occur

Table 4. Lindeque Criteria

1. Sustained $\mathrm{PO}_{2}<8 \mathrm{kPa}$

2. Sustained $\mathrm{PCO}_{2}>7.3 \mathrm{kPa}$

3. Sustained respiratory rate $>35 / \mathrm{min}$, in spite of sedation

4. Increased work of breathing, dyspnea, tachycardia, anxiety

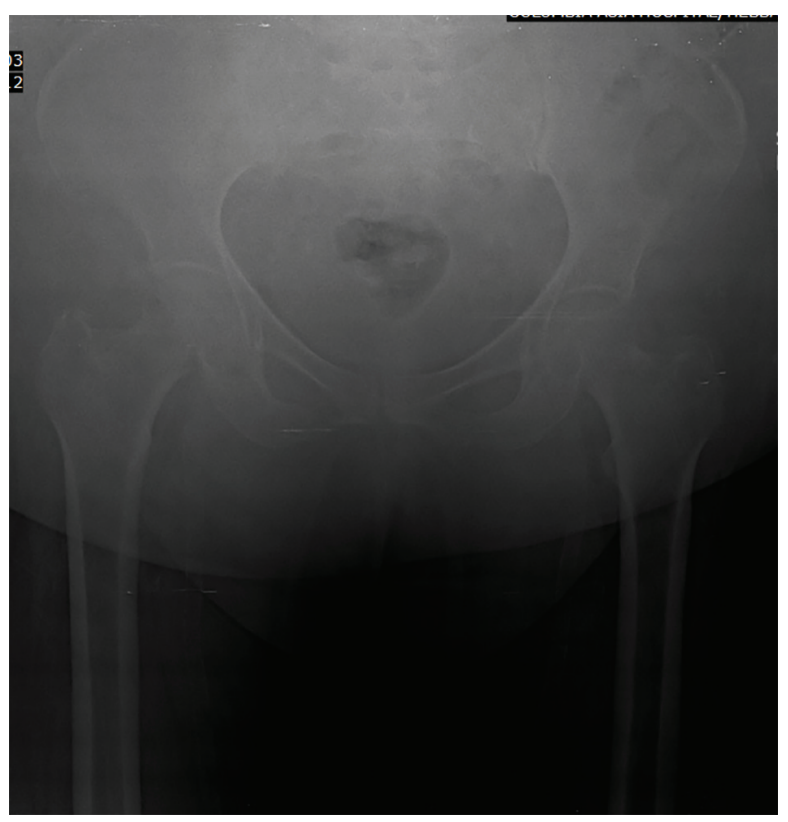

Figure 1. Fracture of right inferior pubic ramus. 


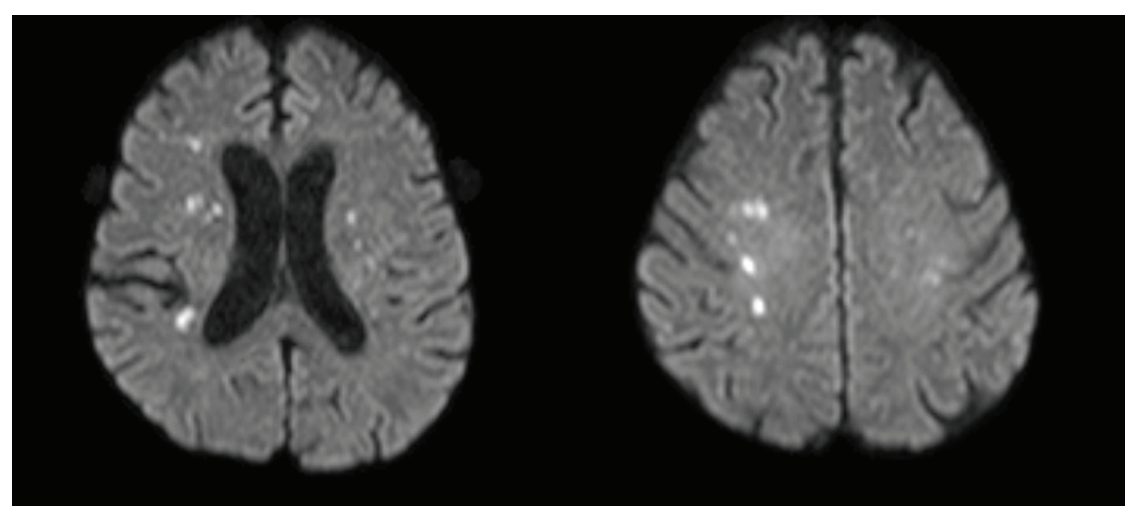

Figure 2. Diffusion weighted image showing fat embolism.

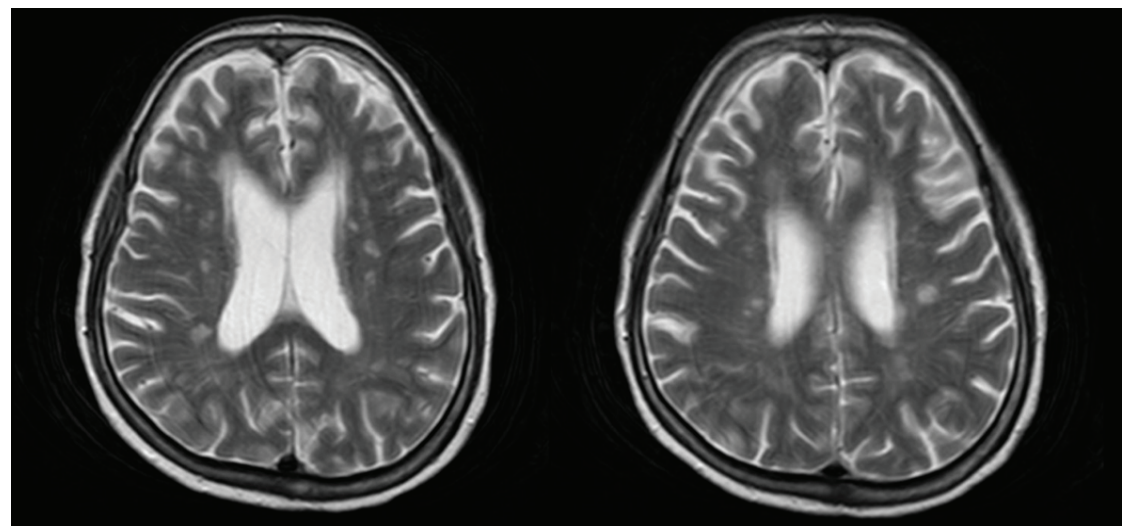

Figure 3. T2-weighted image showing fat embolism.

as subclinical events. Neurological dysfunction in FES varies significantly from confusion to encephalopathy with coma and seizures. Blunt carotid artery injury and vertebrobasilar artery thrombosis also should be considered as the differential diagnosis of non-head-injured patients with acute mental status change.

The neuroradiological diagnosis of cerebral fat embolism is challenging as the brain CT scans are mostly negative. MRI is more sensitive and consistently shows multiple small, scat- tered, hyperintense non-confluent intracerebral lesions on T2weighted scans as reported in our patient. Signal abnormalities can be noticed in both the gray and white matter. Lesions disappear gradually within weeks to a few months.

However, T2-weighted MRI scans are of much help in the (hyper)acute phase. The abnormalities may develop after several days and findings may be non-specific. Differential diagnosis of disseminated hyperintense lesions on T2-weighted scans includes diffuse axonal injury, foci of gliosis, areas of

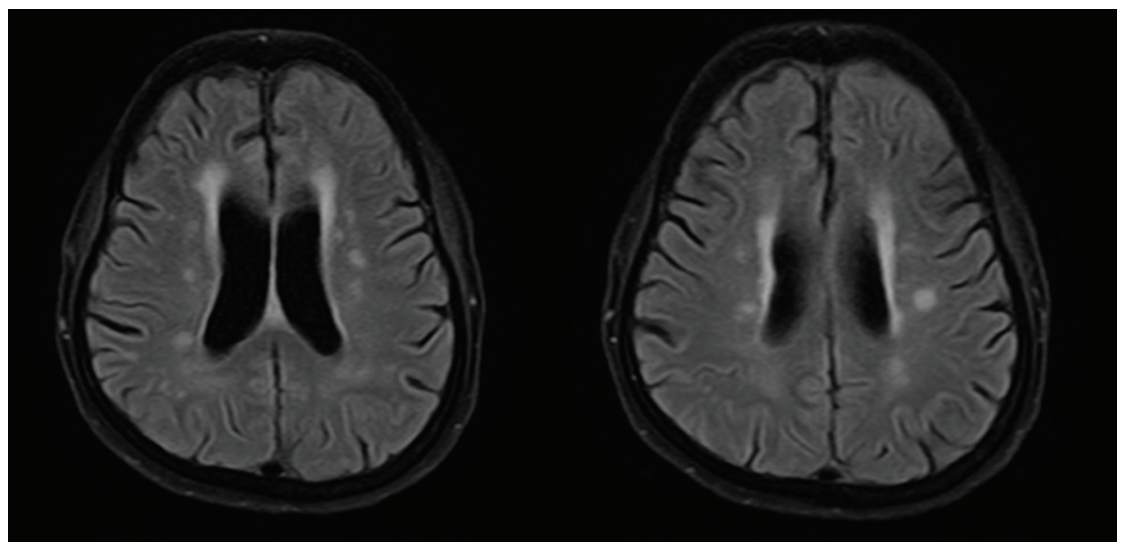

Figure 4. T1 FLAIR image showing fat embolism. 


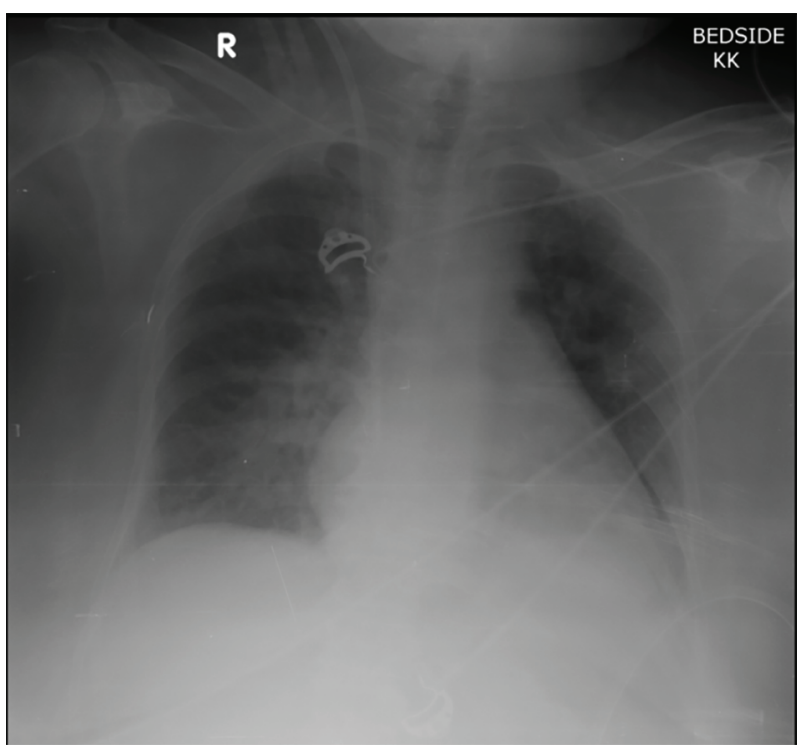

Figure 5. Chest radiograph showing features of ARDS.

vasogenic edema associated with microinfarcts, demyelinating disease and dilated perivascular Virchow-Robin spaces. Our case report showed MRI sensitivity in T2WI, DWI and FLAIR images.

The treatment of FES is only supportive that includes maintenance of adequate oxygenation, hemodynamic stability, hydration, prevention of deep venous thrombosis and gastrointestinal bleeding, and nutrition. High-dose corticosteroids were found to be effective in preventing the development of FES in several studies, but the use remains controversial. Albumin has been recommended as it restores blood levels and combines the fatty acids that may limit lung injury.

In conclusion, FES diagnosis requires high index of suspicion as there are no pathognomonic signs. Treatment of FES is mainly supportive and use of corticosteroids and heparin remains controversial. MRI scan is very useful in diagnosis, DW-MRI reveals the cytotoxic edema and T2-weighted scans reflect vasogenic edema.

\section{Conflicts of Interest}

None.

\section{Funding}

None.

\section{References}

1. Fulde GW, Harrison P. Fat embolism - a review. Arch Emerg Med. 1991;8(4):233-239.

2. Habashi NM, Andrews PL, Scalea TM. Therapeutic aspects of fat embolism syndrome. Injury. 2006;37(Suppl 4):S68-73.

3. Shaikh N. Emergency management of fat embolism syndrome. J Emerg Trauma Shock. 2009;2(1):29-33.

4. Mellor A, Soni N. Fat embolism. Anaesthesia. 2001;56(2) :145-154.

5. Carr JB, Hansen ST. Fulminant fat embolism. Orthopedics. 1990;13(2):258-261.

6. Raman R, Sidhom S, Pape HC, Giannoudis PV. Systemic effects of bilateral tibial versus bilateral femoral shaft fractures. Is there a difference? Acta Orthop Belg. 2004;70(2):134-141.

7. Carlson DS, Pfadt E. Fat embolism syndrome. Nursing. 2011;41(4):72.

8. Schonfeld SA, Ploysongsang Y, DiLisio R, Crissman JD, Miller E, Hammerschmidt DE, Jacob HS. Fat embolism prophylaxis with corticosteroids. A prospective study in high-risk patients. Ann Intern Med. 1983;99(4):438-443.

9. Lindeque BG, Schoeman HS, Dommisse GF, Boeyens MC, Vlok AL. Fat embolism and the fat embolism syndrome. A double-blind therapeutic study. J Bone Joint Surg Br. 1987;69(1):128-131.

10. Muller C, Rahn BA, Pfister U, Meinig RP. The incidence, pathogenesis, diagnosis, and treatment of fat embolism. Orthop Rev. 1994;23(2):107-117. 